\title{
COVID-19 Vaccine Hesitancy Among Patients with Inflammatory Bowel Diseases at a Diverse Safety Net Hospital
}

\author{
Howard S. Herman ${ }^{1}$ (D) Max P. Rosenthaler ${ }^{1} \cdot$ Noon Elhassan $^{2} \cdot$ Janice M. Weinberg $^{2} \cdot$ Venkata R. Satyam $^{3}$. \\ Sharmeel K. Wasan ${ }^{3}$
}

Received: 6 October 2021 / Accepted: 25 January 2022 / Published online: 17 February 2022

(c) The Author(s), under exclusive licence to Springer Science+Business Media, LLC, part of Springer Nature 2022

\begin{abstract}
Background and Aims Patients with inflammatory bowel disease (IBD) and underrepresented minorities (URMs) historically have below average vaccination rates. URMs have increased morbidity and mortality from COVID-19. We surveyed IBD patients to assess COVID vaccination attitudes, particularly among URMs.

Methods In May and June 2021, all 822 adult patients with IBD, medically homed at a tertiary IBD referral center and safety net hospital, and with access to the electronic patient portal, were sent an electronic survey assessing their attitudes regarding COVID-19 vaccination. An additional 115 without access to the patient portal were contacted by phone. Demographic and clinical data were recorded. The primary outcome was vaccination hesitancy, defined as: likely will become vaccinated later this year, but not immediately; unsure if they will get the vaccine; or do not want the vaccine. Multivariable logistic regression was used to calculate adjusted odds ratios (aOR) of factors associated with vaccination intent.

Results The mean age was 46.6 years (SD 15.1). 210/1029 patients responded to the survey: $150 / 822$ (18.2\%) electronically and 60/115 (52.2\%) by phone. Overall vaccine hesitancy rate was $11.9 \%$, significantly higher in younger (aOR for 10-year increments, 0.64 ; 95\% confidence interval [CI], 0.46-0.90, $p=0.011$ ), Hispanic (aOR, 7.67; 95\% CI, 2.99-21.3, $p<0.0002$ ), and Black patients (aOR, 3.52; 95\% CI 1.11-11.1, $p=0.050$ ). Safety concerns were the most cited reasons for vaccine hesitancy.

Conclusions URM patients were more vaccine hesitant. Future studies should further explore factors leading to lower vaccination rates among these groups and strategies to improve COVID-19 vaccination rates.
\end{abstract}

Keywords SARS-CoV-2 $\cdot$ COVID-19 $\cdot$ Vaccine $\cdot$ Vaccine hesitancy $\cdot$ Inflammatory bowel disease $\cdot$ IBD $\cdot$ Underrepresented minorities $\cdot$ Healthcare disparities

Howard S. Herman

howard.herman@bmc.org

Max P. Rosenthaler

mprosent@bu.edu

Noon Elhassan

noon001@bu.edu

Janice M. Weinberg

janicew@bu.edu

Venkata R. Satyam

Venkata.Satyam@bmc.org
Sharmeel K. Wasan

Sharmeel.Wasan@bmc.org

Department of Medicine, Boston Medical Center, Boston, MA, USA

2 Department of Biostatistics, Boston University School of Public Health, Boston, MA, USA

3 Section of Gastroenterology, Department of Medicine, Boston Medical Center, Boston, MA, USA 


\section{Body of Paper}

\section{Background}

The Coronavirus disease 19 (COVID-19) pandemic continues to spread throughout the USA and across the world. Patients with inflammatory bowel disease (IBD) carry the same risk of infection as the general population but may be at increased risk of severe disease [1-4]. Vaccinations were authorized for emergency use in the USA in December 2020 and February 2021, with full approval for one issued in August 2021. The International Organization for the Study of Inflammatory Bowel Diseases recommends COVID-19 vaccination for all adults with IBD [5]. In a recent survey of patients with IBD, $81 \%$ of respondents received or were willing to receive a COVID-19 vaccine [6]. However, $89 \%$ had high educational attainment and 93\% identified as White, while less than 5\% were Black or of Hispanic ethnicity. In contrast, underrepresented minorities (URMs) historically have had lower vaccination rates due to higher prevalence of mistrust of healthcare institutions [7-14]. Notably, the CDC reported COVID vaccination rates from December 2020 to May 2021 were lower among socially vulnerable Americans such as nonHispanic White persons, uninsured individuals, or people living in poverty [15]. Among patients with IBD, historical vaccination rates have also been below average, possibly due to concerns about vaccine efficacy and safety in patients on immunosuppressants [16-18]. URMs are also known to have higher rates of morbidity and mortality from COVID-19 [19]. Such data suggest that URM patients with IBD will be among the most hesitant to receive the COVID-19 vaccine and consequently the most vulnerable to poor outcomes. To date, the rate of COVID19 vaccine hesitancy among URM patients with IBD is unknown.

\section{Methods}

Patients were recruited from a local population of 1265 adult patients medically homed at the Boston Medical Center (Boston, MA) a tertiary IBD referral center and safety net hospital, identified by diagnosis codes for Crohn's disease (ICD-10-CM K50), ulcerative colitis (ICD-10-CM K51), or indeterminate colitis (ICD-10-CM K52.3) obtained from the electronic medical record.

In May and June 2021, while the COVID-19 vaccines were under Emergency Use Authorization by the FDA, 822 patients were sent an electronic survey assessing their attitudes regarding COVID-19 vaccination via the electronic medical record's patient portal, MyChart (Epic Systems Corp., Verona, WI). A total of 207 patients without access to the patient portal were contacted to complete the survey by phone.

Respondents were not provided reimbursement or other incentivization for completing the survey. Demographic and clinical data were abstracted from the electronic medical record (EMR). Study data were collected and managed using REDCap electronic data capture tools hosted at Boston University, CTSI 1UL1TR001430.19 [20]. The primary outcome was vaccination hesitancy, defined as: likely will become vaccinated later this year, but not immediately; unsure if they will get the vaccine; or do not want the vaccine. Chi-squared analyses were performed to determine which variables were most likely to be significant for multivariable logistic regression. Multivariable logistic regression was then used to calculate adjusted odds ratios (aORs) of factors associated with vaccination intent. Odds ratios along with $95 \%$ confidence intervals are reported. Analyses were performed using $\mathrm{R}$ with $p<0.05$ considered statistically significant. This study was approved by the Institutional Review Board of Boston University Medical Campus and Boston Medical Center.

\section{Results}

Two hundred and ten of out 1029 participants completed the survey, with 150 responding electronically and 60 by phone. The electronic response rate was $150 / 822(18.2 \%)$. Due to staffing limitations and the urgency of the pandemic, 207 of the 433 patients without access to MyChart were contacted by phone to complete the survey, of whom 60 (52.2\%) completed the survey, 55 declined to participate, and 92 were not reached. The mean age was 46.6 years (standard deviation, 15.1).

Demographic and IBD characteristics are presented in Table 1 . The overall vaccine hesitancy rate was $11.9 \%$. Vaccine hesitancy was significantly higher in younger patients (aOR for 10-year increments, $0.64 ; 95 \%$ confidence interval $[\mathrm{CI}], 0.46-0.90, p=0.011)$, Hispanic patients $(27.8 \%$ vs. 6.4\%, aOR, 7.67; 95\% CI, 2.99-21.3, $p<0.0002$ ), and Black patients $(15.9 \%$ vs. $11.3 \%$, aOR, 3.52; 95\% CI $1.11-11.1$, $p=0.050$ ). Out of the Black patients, $79.5 \%$ had already received the vaccine, $4.5 \%$ "wanted to as soon as possible," 9.1\% said "not right away, but likely later in the year," $4.5 \%$ were "undecided," and $2.3 \%$ said "no." Out of the Hispanic patients, $68.5 \%$ had already received the vaccine, $3.7 \%$ "wanted to as soon as possible," $18.5 \%$ said "not right away, but likely later in the year," 5.6\% were "undecided," and $3.7 \%$ said "no." COVID-19 vaccine attitudes are presented in Tables 2 and 3. Patients not on biologics or immunomodulators $(15.1 \%$ vs. $9.6 \%)$ and patients with lower educational 
Table 1 Patient characteristics

\begin{tabular}{ll}
\hline & $N=210(\%)$ \\
\hline Age (years, mean \pm SD) & $46.6 \pm 15.1$ \\
Male & $76(36.2 \%)$ \\
Hispanic & $54(25.7 \%)$ \\
Race & \\
White & $160(76.2 \%)$ \\
Black & $44(21.0 \%)$ \\
Asian Pacific Islander or Native Hawaiian & $5(2.4 \%)$ \\
Other & $1(0.48 \%)$ \\
Education Level & \\
No high school degree & $35(16.7 \%)$ \\
High school graduate or GERD & $51(24.3 \%)$ \\
Some college, vocational, or technical school & $30(14.3 \%)$ \\
Graduated college & $47(22.4 \%)$ \\
Not specified & $47(22.4 \%)$ \\
Primary Language & \\
English & $165(78.6 \%)$ \\
Spanish & $44(21.0)$ \\
Cape Verdean/Port Creole & $1(0.48 \%)$ \\
Biologics or Immunomodulators & $104(49.5 \%)$ \\
Vaccinated for Influenza & $154(73.3 \%)$ \\
\hline
\end{tabular}

attainment ( $14.1 \%$ vs. $4.3 \%$ ) tended to have higher rates of hesitancy, but these comparisons were not statistically significant. The most common reasons for vaccine hesitancy were "concerns about adverse reactions" (72\%), "the vaccine didn't undergo necessary scrutiny and safety checks" (64\%), and "the long-term safety of the COVID vaccine is unknown" (52\%).

\section{Discussion}

COVID vaccination rates in parts of the USA continue to trail stated targets [21]. Our study demonstrates a lower overall rate of COVID-19 hesitancy compared to the prior study of patients with IBD (11.9\% vs. 19\%) [6], though the prior study was performed when vaccines were first approved for emergency use, whereas our study occurred months later, with many patients already vaccinated. Encouragingly, both populations had lower rates of COVID-19 vaccine hesitancy than the country overall, in which up to $30 \%$ of adults remain vaccine hesitant [22]. The above represents a reversal of previous studies for other vaccines in IBD patients [16-18], the reasons for which are not immediately clear.
Table 2 COVID-19 vaccine attitudes

\begin{tabular}{ll}
\hline & $N=210(\%)$ \\
\hline Not Vaccine Hesitant & $185(88.10 \%)$ \\
Already vaccinated & $179(85.23 \%)$ \\
Want to be vaccinated as soon as possible & $6(2.86 \%)$ \\
Vaccine Hesitant & $25(11.90 \%)$ \\
Likely will become vaccinated later this year, but not immediately & $12(5.71 \%)$ \\
Unsure if they will get the vaccine & $7(3.33 \%)$ \\
Do not want the vaccine & $6(2.86 \%)$ \\
Reasons for Vaccine Hesitancy & \\
Concerned about adverse reaction & $18(72 \%)$ \\
Concerned vaccine could interfere with IBD medication efficacy & $11(44 \%)$ \\
Concerned IBD med might make vaccination ineffective & $9(36 \%)$ \\
Already had COVID & $3(12 \%)$ \\
Negative experiences with last vaccine & $2(8 \%)$ \\
Generally don't take vaccines & $5(20 \%)$ \\
Long-term safety of the COVID vaccine is unknown & $13(52 \%)$ \\
Concerned vaccine didn't undergo necessary scrutiny and safety checks & $16(64 \%)$ \\
Personal history of allergic reaction & $1(4 \%)$ \\
Prefer to watch how others tolerate the vaccine & $12(48 \%)$ \\
What can IBD providers do to better inform you about COVID vaccines? & \\
Have a risk/benefit conversation about it & $11(44 \%)$ \\
Provide handout information about the vaccine & $8(32 \%)$ \\
Provide data about the efficacy among patients withIBD and other immune diseases & $10(40 \%)$ \\
Nothing & $9(36 \%)$ \\
Unsure & $11(44 \%)$ \\
\hline & $2(8 \%)$ \\
\hline
\end{tabular}


Table 3 Univariate and multivariate analyses

\begin{tabular}{lll}
\hline & $\%$ (fraction) & $\mathrm{OR}^{\mathrm{b}}(95 \% \mathrm{CI})$ \\
\hline $\begin{array}{l}\text { Vaccine Hesitant } \\
\text { Age }{ }^{*}\end{array}$ & $11.9 \%(25 / 210)$ & $0.64^{\mathrm{c}}(0.46,0.90)$ \\
Vaccine Hesitant & 40.20 & \\
Not Hesitant & 47.55 & $7.67(2.99,21.3)$ \\
Hesitancy by Ethnicity*** & & \\
Hispanic & $27.8 \%(15 / 54)$ & $3.52(1.12,11.1)$ \\
Not Hispanic & $6.4 \%(10 / 156)$ & \\
Hesitancy by Race* & & \\
Black & $15.9 \%(7 / 44)$ & \\
White & $11.3 \%(18 / 160)$ & \\
Hesitancy by Education & & \\
Associate's or lower & $14.1 \%(23 / 163)$ & \\
Bachelor's or higher & $4.3 \%(2 / 47)$ & \\
Hesitancy by Treatment Regimen & $15.1 \%(15 / 106)$ & \\
Taking Biologic or Immunomodulator & $9.6 \%(10 / 104)$ & \\
Not Taking Biologic or Immunomodulator & & \\
\hline
\end{tabular}

Multivariate regression including age, ethnicity, race, and education

${ }^{\mathrm{a}}$ Mean age, years

${ }^{\mathrm{b}} \mathrm{OR}$ : odds ratio

${ }^{\mathrm{c}}$ Odds ratio for ten-year age differences

${ }^{*} p<0.05$ in univariate and multivariate analyses

${ }^{* * *} p<0.00001$ in univariate and multivariate analyses
In our population, a relatively higher rate of COVID-19 vaccine hesitancy was observed among younger, Hispanic, and Black patients. Participants with lower educational attainment and those on biologics or immunomodulators also trended toward higher rates of hesitancy. The results are concordant with previous studies that showed higher vaccine hesitancy among URM patients [7-14]. Reasons for COVID-19 vaccine hesitancy among URM patients with IBD appear to mirror those seen in non-IBD patients that self-identify with these groups. This distrust in healthcare institutions is due to current or prior negative experiences with health care providers, suspicion about prior racist study practices, belief that minority groups are underrepresented in validating research, and many other complex social and environmental factors.

Study strengths include a diverse study population. Limitations include small sample size, survey response bias, and low response rate that may overestimate vaccination intent.

Our study demonstrates higher rates of vaccine hesitancy among our URM population, mainly due to concerns about long-term safety. IBD providers should educate and advocate for their URM patients to ensure high uptake of COVID-19 vaccination among all their patients. A recent review provided a useful framework for addressing vaccine hesitancy, which employs an empathetic, informative, and decisive approach. Providers should validate concerns, reassuring that hesitancy is common; they should discuss the risks and benefits, using positive framing to emphasize the benefits of vaccination; and they should strongly recommend getting immunized [23]. The CDC also offers toolkits to help allay concerns and misconceptions about COVID-19 vaccines [24]. Future studies should explore the efficacy of various approaches to increasing COVID-19 vaccination, as well as other possible factors that lead to lower vaccination rates among these groups.

Supplementary Information The online version contains supplementary material available at https://doi.org/10.1007/s10620-022-07413-y.

Funding This publication was supported by the National Center for Advancing Translational Sciences, National Institutes of Health, through Boston University Clinical \& Translational Science Institute Grant Number 1UL1TR001430. Its contents are solely the responsibility of the authors and do not necessarily represent the official views of the NIH.

\section{Declarations}

Conflict of interest The authors declare that they have no conflict of interest.

\section{References}

1. Khan N, Patel D, Xie D et al. Are patients with inflammatory bowel disease at an increased risk of developing SARS-CoV-2 
than patients without inflammatory bowel disease? Results from a nationwide Veterans' Affairs cohort study. Am J Gastroenterol. 2021;116:808-810.

2. Brenner EJ, Ungaro RC, Gearry RB et al. Corticosteroids, but not TNF antagonists, are associated with adverse COVID-19 outcomes in patients with inflammatory bowel diseases: results from an international registry. Gastroenterology. 2020;159:481-491.

3. Ungaro RC, Brenner EJ, Gearry RB et al. Effect of IBD medications on COVID-19 outcomes: results from an international registry. Gut. 2021;70:725-773.

4. Derikx LAAP, Lantinga MA, de Jong DJ et al. Clinical outcomes of Covid-19 in patients with inflammatory bowel disease: a nationwide cohort study. J Crohns Colitis. 2021;15:529-539.

5. Siegel CA, Melmed GY, McGovern DP, on behalf of the International Organization for the Study of Inflammatory Bowel Disease (IOIBD) et al. SARS-CoV-2 vaccination for patients with inflammatory bowel diseases: recommendations from an international consensus meeting. Gut. 2021;70:635-640.

6. Dalal RS, McClure E, Marcus J et al. COVID-19 vaccination intent and perceptions among patients with inflammatory bowel diseases. Clin Gastroenterol Hepatol. 2021;19:1730-1732.

7. Freimuth VS, Jamison AM, An J et al. Determinants of trust in the flu vaccine for African Americans and Whites. Soc Sci Med. 2017;193:70-79.

8. Fu LY, Zimet GD, Latkin CA et al. Associations of trust and healthcare provider advice with HPV vaccine acceptance among African American parents. Vaccine. 2017;35:802-807.

9. Kolar SK, Wheldon C, Hernandez ND et al. Human papillomavirus vaccine knowledge and attitudes, preventative health behaviors, and medical mistrust among a racially and ethnically diverse sample of college women. J Racial Ethn Health Dispar. 2015;2:77-85.

10. Lee C, Whetten K, Omer S et al. Hurdles to herd immunity: distrust of government and vaccine refusal in the US, 2002-2003. Vaccine. 2016;34:3972-3978.

11. Quinn SC, Jamison A, Freimuth VS et al. Exploring racial influences on flu vaccine attitudes and behavior: results of a national survey of White and African American adults. Vaccine. 2017;35:1167-1174.

12. Harris LM, Chin NP, Fiscella K, Humiston S. Barrier to pneumococcal and influenza vaccinations in Black elderly communities: mistrust. J Nat Med Assoc. 2006;98:1678.

13. Quinn S, Jamison A, Musa D, et al. Exploring the continuum of vaccine hesitancy between African American and white adults: results of a qualitative study. PloS Curr. 2016;8.

14. Freimuth VS, Jamison A, Hancock G et al. The role of risk perception in flu vaccine behavior among African American and white adults in the United States. Risk Anal. 2017;37:2150-2163.
15. Diesel J, Sterrett N, Dasgupta S et al. COVID-19 vaccination coverage among adults - United States, December 14, 2020-May 22, 2021. MMWR Morb Mortal Wkly Rep. 2021;70:922-927.

16. Malhi G, Rumman A, Thanabalan $R$ et al. Vaccination in inflammatory bowel disease patients: attitudes, knowledge, and Uptake. J Crohn's Colitis. 2015;6:439-444.

17. Alexander JL, Moran GW, Gaya DR, On behalf of the Inflammatory Bowel Disease section of the British Society of Gastroenterology and the Inflammatory Bowel Disease Clinical Research Group et al. SARS-CoV-2 vaccination for patients with inflammatory bowel disease: a British society of gastroenterology inflammatory bowel disease section and IBD clinical research group position statement. Lancet Gastroenterol Hepatol. 2021;6:218-224.

18. Chan W, Salazar E, Lim TG et al. Vaccinations and inflammatory bowel disease - a systematic review. Dig Liver Dis. 2021;53:1079-1088.

19. Ndugga N, Hill L, Artiga S Parker N. Latest Data on COVID19 Vaccinations by Race/Ethnicity. Available at https://www.kff. org/coronavirus-covid-19/issue-brief/latestdata-on-covid-19-vacci nations-race-ethnicity/. Published 18 Aug 2021, Accessed 9.08.21

20. Harris P, Taylor R, Thielke R et al. Research electronic data capture (REDCap) - A metadata-driven methodology and workflow process for providing translational research informatics support. J Biomed Inform. 2009;42:377-381.

21. Bebinher M, Farmer B. As COVID Vaccinations Slow, Parts of The U.S. Remain Far Behind 70\% Goal. NPR. Available at https:// www.npr.org/sections/healthshots/2021/07/05/1013080128/ascovid-vaccinations-slow-parts-of-the-u-s-remain-farbehind-70goal. Published 5 Jul 2021, Accessed 3.09.21.

22. Kirzinger A, Sparks G, Hamel K, Lopes L, Kearney A, Stokes M, Brodie M. KFF COVID-19 Vaccine Monitor: July 2021. Available at https://www.kff.org/coronaviruscovid-19/poll-finding/ kff-covid-19-vaccine-monitor-july-2021/. Published 4 Aug 2021, Accessed 13.09.21.

23. Caldera F, Balzora S, Hayney $\mathrm{M}$ et al. Ensuring high and equitable COVID-19 vaccine uptake among patients with IBD. Inflamm Bowel Dis. 2021. https://doi.org/10.1093/ibd/izab114.

24. Center for Disease Control and Prevention. (CDC) Vaccinate with confidence. Strategies to reinforce confidence in COVID-19 vaccines. Available at https://www.cdc.gov/vaccines/covid-19/vacci nate-with-confidence.html. Accessed 13.12.21.

Publisher's Note Springer Nature remains neutral with regard to jurisdictional claims in published maps and institutional affiliations. 